\title{
Diagnostic challenge - West Nile encephalitis mimicking stroke - case report
}

\author{
Maria-Gabriela Catana ${ }^{1,2}$, Liliana-Cristina Cuibus', Ionela-Alexandra Cosma', \\ Corina Roman-Filip ${ }^{1,2}$ \\ ${ }^{1}$ Department of Neurology, Emergency Clinical County Hospital, Sibiu, Romania \\ ${ }^{2}$ Faculty of Medicine, "Lucian Blaga" University, Sibiu, Romania
}

\begin{abstract}
West Nile virus (WNV) is widely distributed around the world. The WNV is maintained in nature by a cycle involving mosquitoes and birds, which are presumed to be the most important amplifying hosts. We present the case of a 75 years old male patient admitted to the neurology department for several symptoms that could be attributed to a stroke. The symptomatology had an acute onset. The computed tomography and the magnetic resonance imaging revealed lacunar stroke in right semioval center. The final diagnosis was established by the lumbar punction: West Nile virus was highlighted in the cerebrospinal fluid, clinical evolution was favorable, the patient was discharge with minimal neurologic deficits.
\end{abstract}

Keywords: stroke, West Nile virus, stroke mimics, encephalitis

\section{BACKGROUND}

West Nile virus (WNV) is widely distributed around the world. The WNV is maintained in nature by a cycle involving mosquitoes and birds, which are presumed to be the most important amplifying hosts (1). Suspected outbreaks of WNV infection have been reported in Romania since the 1950s. The incubation period for clinical illness generally ranges from 2 to 14 days. West Nile fever can range from a mild infirmity lasting a few days to a debilitating illness lasting weeks to months. Symptoms are of sudden onset and often include headache, malaise, fever, myalgia, chills, vomiting, rash, fatigue, and eye pain (2). Fever may be lowgrade or absent. West Nile encephalitis ranges in severity from a mild, self-limited confusional state to severe encephalopathy, coma, and death (3).

\section{CASE PRESENTATION}

We present the case of a 75 years old male patient, known with a medical history of stroke - mild left hemiparesis 4/5 MRC (medical research council) - Rankin 1 (ischemic stroke in the right cerebral media artery) and carotid atheromatosis, who was admitted to the neurology department for right oculocephalic deviation, left central facial palsy, left hemiplegia and dysarthria, symptomatology with an acute onset. The neurological exam revealed additionally, bilateral plantar extensor response (Babinski sign), no coordination and sensitivity problems. The patient was hemodynamic stable: rhythmic heart rate, without significant pulmonary pathology, and no fever. The electrocardiogram was in normal limits. National Institutes of Health Stroke Scale (NIHSS) summed up 14 points: forced oculocephalic deviation (2), complete hemianopia (2), minor paralysis (flattened nasolabial fold, asymmetry on smiling) (1), no movements of the left arm (4) and left leg (4), mild-to-moderate dysarthria (1). Due to the fact that the patient was admitted to the neurology department after more than 4.5 hours from the time of the symptomatology`s onset thrombolysis was not taken into account. 
At admission the patient presented no comatose state - 15 points on Glasgow Coma Scale (GCS).

Cerebral unenhanced computed tomography (CT), perfomed at admission in emergency department, revealed lacunar strokes in the right semioval center. Doppler ultrasounds excluded significant stenosis or occlusion.

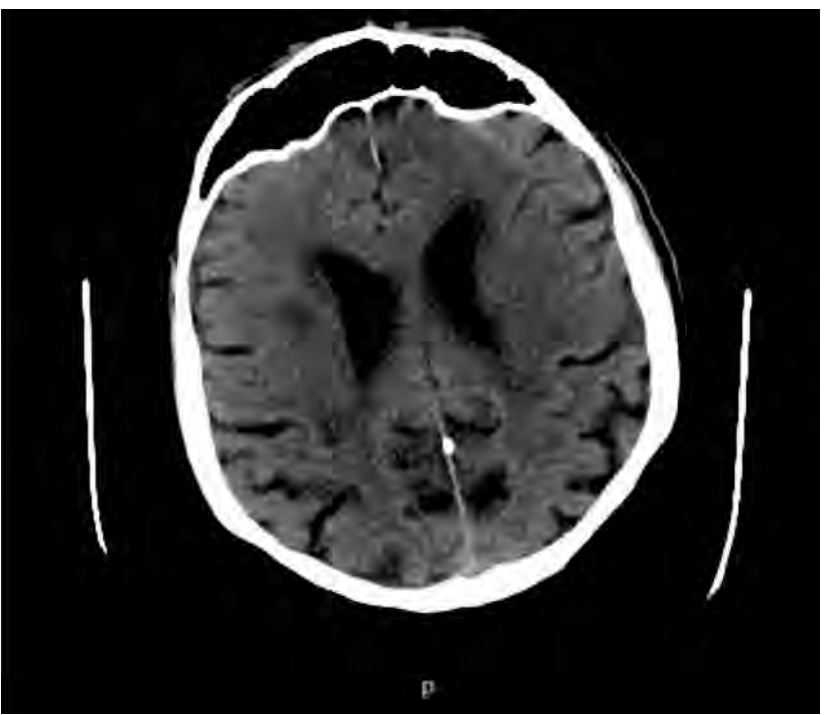

FIGURE 1. Cerebral CT - Lacunar stroke in the right centrum semiovale

After 24 hours the patient's condition worsened: he had a comatose state -8 points GCS and developed fever $-39^{\circ} \mathrm{C}$. The second native cranio-cerebral computed tomography examination performed,had the same result as the first one. The Cerebral MRI revealed same lacunar strokes in the right semioval center. The cerebrospinal fluid (CSF) analysis revealed increased total protein $2,479 \mathrm{~g} / 1$ - (normal range $0,15-0,45 \mathrm{~g} / \mathrm{l}$ ) with 140 elements, predominant polymorphonuclear cells. Samples from CSF were sent to the public health department for further lab tests, virus and bacteriological screening. Considering the clinical, radiological and biological features, acute meningoencephalities was suspected.

Due to clinical evolution, the patient was intubated and connected to mechanic ventilation in the ICU department, for 5 days. The infectious disease physician, decided that the patient should be treated with antiviral (Acyclovir) and antibiotic therapy (Cefort), according to infectious diseases` guidelines.

Few days after the treatment was initiated, the patient's consciousness level improved. He started feeling better and at 10 days from admission he was discharged.

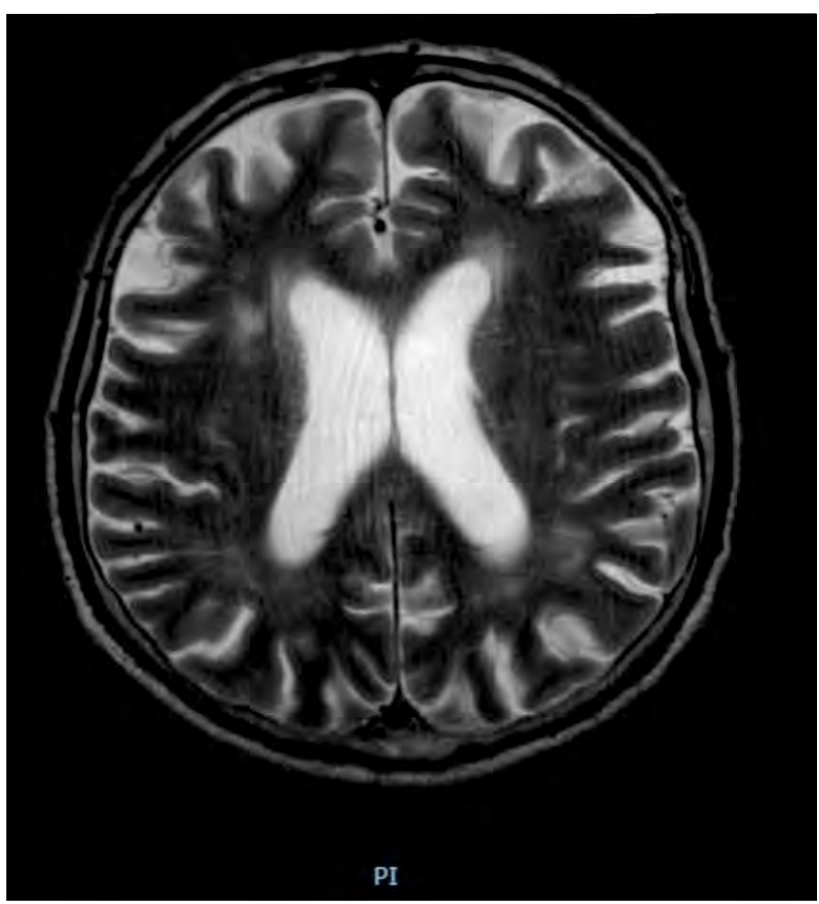

FIGURE 2. Cerebral MRI - T2 weighted sequence - Lacunar stroke in the right centrum semiovale

At discharge the neurological exam revealed: the patient can move alone, with unilateral support and presents a left hemiparesis with grade $4 / 5$ power (Medical Research Council grading) in left upper and lower limbs, else, in normal limits, Rankin Scale 1.

\section{DISCUSSION}

Stroke remains one of the most important causes of disability and death worldwide (4). Prompt recognition of an acute ischemic stroke is crucial, as prior imaging-based work has demonstrated that the volume of irreversibly damaged brain tissue expands rapidly until reperfusion occurs (5). Rapid recognition followed by early thrombolytic treatment was associated with improved outcome. But, however, timely recognition and treatment is complicated by the fact that there are multiple conditions that mimic acute ischemic stroke. In various studies, the most common stroke mimics include brain tumors (6), toxic or metabolic disorders (7), infectious disorders (e.g. meningoencephalitis), conversion/ functional (psychiatric disorder), migraines, seizures and demyelization disorders (8). But, there are no specific clinical signs and/or symptoms to identify stroke mimics. 
In the presented case, the patient was admitted to the Neurology department with the hypothesis of acute stroke. Because, the patient had a history of ischemic right carotidian stroke and carotid atheromatosis the first thought was that the patient had a recurrent stroke. Even in this case, it was once again strongly emphasized the fact that the brain imaging is essential for the correct diagnosis of acute ischemic stroke and stroke mimic exclusion.

It is known the fact that sometimes the MRI can reveal no acute pathology in WNV meningoencephalitis. Studies have shown that patients with normal MR images or abnormalities on only DW images have the best prognosis (3).

The primary purpose of neuroimaging in a patient with suspected ischemic stroke is to rule out the presence of other types of central nervous system lesions and to distinguish between ischemic and hemorrhagic stroke (9). CT scans are considered sufficiently sensitive for detecting mass lesions, such as a brain mass or abscess, as well as detecting acute hemorrhage. The purpose of a CT scan is to exclude unidentified strokes mimics and detect haemorrhage. In essence, a normal CT scan does not exclude the suspicion of an acute ischemic stroke, or some stroke mimics like meningoencephalitis (10).

In the presented case, the CT exam excluded an acute pathology of stroke as well some other pathologies (brain mass or abscess, acute hemorrhage) that can imitate a stroke.

After a detailed anamnesis, we excluded a history of migraine (history of similar events, preceding aura and headache), a possible seizure (history of loss consciousness, seizure activity), a psychiatric disorder (presence of known cognitive impairment) a possible alcohol intoxication, medication adverse effect, which could mimic a stroke. Also, toxic or metabolic disorders, hypoglycemia most common, was excluded $(11,12)$.

The particularity of this case was that the patient had no fever, no headache, no seizures and at presentation in emergency room all the patient's symptoms suggested a stroke. The fact that the patient had no fever made it difficult to decide the presumptive diagnosis. Due to the fact that the patient's condition worsened $24 \mathrm{~h}$ later, developing fever with a temperature of $39^{\circ} \mathrm{C}$, led us to the diagnosis. The first CSF analysis before receiving the
WNV confirmation suggested encephalitis. So, the next step was to establish the aetiology of encephalitis and the treatment to be initiated.

In most common studies, the characteristic presentation of viral encephalitis usually consists of fever, headache, and clouding of consciousness together with seizures and focal neurological signs, in some cases.

However, the distinction between infective viral encephalitis and a metabolic encephalopathy or acute disseminated encephalitis may not always be straightforward. Once a diagnosis of infective viral encephalitis has been established it is then necessary to have a clear investigative plan to try to determine the likely cause (13).

It is important another causes of viral encephalitis: herpes simplex virus, adenovirus, enterovirus and arbovirus (for example, Japanese B encephalitis, St Louis encephalitis virus, West Nile encephalitis virus, Eastern, Western, and Venezuelan equine encephalitis virus, tick borne encephalitis viruses) to be considered. The fact that the diagnosis cannot be certain from the first contact with the patient, there are a number of clues that could help us. So it's important to know about a history of recent travel, a history of recent animal bites, a recent contact with infectious conditions, the occupation of the patient, the season, or any predisposing factors which should also be noted, such as immunosuppression caused by disease and/or drug treatment $(14,15)$. In this case, it's very useful to highlight that the patient was a shepherd and it was summer - mosquitos` favourable environment.

So, it`s very important to make a correct and fast differential diagnosis because West Nile encephalitis ranges in severity from a mild, self-limited confusional state to severe encephalopathy, coma, and death.

\section{CONCLUSIONS}

There is no established antiviral treatment for West Nile encephalitis. Treatment is generally supportive, and no vaccine is currently available for prevention. Humans cannot pass on the disease, so the best prevention is to avoid being bitten by mosquitos. Supportive treatment for patients with West Nile encephalitis includes attention to the complications of infections such as respiratory paralysis, 
pneumonia, pressure sores, and seizures, usually in an intensive care setting.

With this case report we want to point out that is very important West Nile virus to be taken into ac-

\section{REFERENCES}

1. Hayes EB, Komar N, Nasci RS. Epidemiology and transmission dynamics of West Nile virus disease. Emerg Infect Dis. 2005; 11:1167-1173.

2. Pealer LN, Marfin AA, Petersen LR et al. Transmission of West Nile virus through blood transfusion in the United States in 2002. N Engl J Med. 2003; 349(13):1236-1245.

3. Sejvar JJ, Haddad MB, Tierney BC et al. Neurologic manifestations and outcome of West Nile virus infection. JAMA. 2003; 290(4):511-515.

4. M. Edip Gurol and Jong S. Kim. Advances in Stroke Prevention in 2018. J Stroke. 2018 May; 20(2): 143-144.

5. Saver JL. Time is brain - quantified. Stroke 2006; 37:263-266.

6. Hatzitolios A, Savopoulos C, Ntaios G et al. Stroke and conditions that mimic it: A protocol secures a safe early recognition. Hippokratia. 2008; 12:98-102.

7. Demaerschalk BM. Diagnosis and management of stroke (brain attack). Semin Neurol. 2003; 23:241-52.

8. Yew KS, Cheng EM. Diagnosis of acute stroke. Am Fam Physician. 2015; 91:528-36.

9. Kenneth S. Yew et al. Acute Stroke Diagnosis. Am Fam Physician. Author manuscript; available in PMC 2009 Aug 7.Published in final edited form as: Am Fam Physician. 2009 Jul 1; 80(1): 33-40.

10. Mullins ME, Schaefer PW, Sorensen AG et al. CT and conventional and diffusion-weighted MR imaging in acute stroke: Study in 691 count and to be tested in patients with meningoencephalitis.

Conflict of interest: none declared Financial support: none declared

patients at presentation to the emergency department. Radiology. 2002; 224(2):353-360.

11. Nor AM, Davis J, Sen B et al. The Recognition of Stroke in the Emergency Room (ROSIER) scale: Development and validation of a stroke recognition instrument. Lancet Neurol. 2005; 4(11):727-734.

12. Adams HP, Jr, del Zoppo G, Alberts MJ et al. Guidelines for the early management of adults with ischemic stroke: A guideline from the American Heart Association/American Stroke Association Stroke Council, Clinical Cardiology Council, Cardiovascular Radiology and Intervention Council, and the Atherosclerotic Peripheral Vascular Disease and Quality of Care Outcomes in Research Interdisciplinary Working Groups: the American Academy of Neurology affirms the value of this guideline as an educational tool for neurologists. Circulation. 2007 May 22; 115(20):e478-534.

13. Kennedy PG. Viral encephalitis: Causes, differential diagnosis, and management. J Neurol Neurosurg Psychiatry. 2004 Mar; 75 Suppl 1:i10-5.

14. Coyle PK. Postinfectious encephalomyelitis. In: Davis LE, Kennedy PGE, eds. Infectious diseases of the nervous system,1st ed. Butterworth-Heinemann 2000:83-108.

15. Baringer JR. Herpes simplex virus encephalitis. In: Davis LE, Kennedy PGE, eds. Infectious diseases of the nervous system,1st ed. Butterworth-Heinemann, 2002:139-64. 